\title{
AVALIAÇÃO DA DOR DO RECÉM-NASCIDO PELA EQUIPE DE ENFERMAGEM: SCOPING REVIEW
}

\section{EVALUATION OF NEWBORN PAIN BY NURSING TEAM: SCOPING REVIEW}

\section{EVALUACIÓN DEL DOLOR DEL RECIÉN NACIDO POR EL EQUIPO DE ENFERMADO: SCOPING REVIEW}

\author{
Adalvane Nobres Damaceno ${ }^{1}$, Priscila Kurz Assumpção ${ }^{2}$, Graciele Pozzobon da Silva Belmonte ${ }^{3}$
}

\begin{abstract}
RESUMO
Objetivo: identificar as ações da equipe enfermagem na avaliação e manejo da dor no recémnascido. Método: Trata-se de uma Scoping review, realizada em agosto de 2016, mediante busca nas bases de dados Latino-Americana e do Caribe em Ciências da Saúde e Base de Dados da Enfermagem. Foram selecionados oito artigos que atenderam aos critérios de inclusão. Resultados: Identificaram-se estudos transversais, exploratórios qualitativos e quantitativos. Os resultados evidenciaram duas categorias, sendo elas: "A percepção dos profissionais de enfermagem frente ao conhecimento da dor neonatal, os meios de avaliação e a utilização de escalas de dor" a qual foi possível comprovar que os profissionais de enfermagem reconhecem que os recém-nascidos são capazes de sentir dor, as formas de avaliar por meio de aspectos comportamentais e fisiológicos e a existência de escalas para medir dor. Como segunda categoria "Medidas não farmacológicas utilizadas no manejo da dor neonatal" que contemplam a prescrição de fármacos e conduta de manuseio/posicionamento. Conclusão: Conclui-se que ainda há uma grande distância entre o conhecimento teórico, o uso de escalas e a conduta prática dos profissionais em relação à avaliação e manejo da dor neonatal.
\end{abstract}

Descritores: Dor, percepção da dor, recém-nascido, enfermagem

\begin{abstract}
Objective: to identify the actions of the nursing team in the evaluation and management of pain in the newborn. Method: This is a Scoping review, carried out in August 2016, by searching the Latin American and Caribbean databases on Health Sciences and Nursing Database. Eight articles were selected that met the inclusion criteria. Results: Cross-sectional, qualitative and quantitative studies were identified. The results showed two categories: "The perception of nursing professionals regarding the knowledge of neonatal pain, the means of evaluation and the use of pain scales", which confirmed that nursing professionals recognize that newborns are
\end{abstract}

\footnotetext{
${ }^{1}$ Doutorando na Escola de Enfermagem da UFRGS (EENF-UFRGS). Integra projeto com a University British Columbia (UBC - Canadá) com fomento Canadian Institutes of Health Research (CIHR - Canadá) Mestre em Enfermagem pela UFSM. Graduação em Enfermagem pela UFSM. Especialista em Gestão de Organização Pública em Saúde pela UFSM.

2 Mestra em Saúde da Criança PUCRS.

${ }^{3}$ Bacharel em Enfermagem
} 
able to feel pain, the ways to evaluate by behavioral and physiological aspects and the existence of scales to measure pain. As a second category, "Non-pharmacological measures used in the management of neonatal pain" that contemplate the prescription of drugs and handling / positioning behavior. Conclusion: It is concluded that there is still a great distance between the theoretical knowledge, the use of scales and the practitioner's practical conduct in relation to the evaluation and management of neonatal pain.

Descriptors: Pain, pain perception, newborn, nursing

\section{RESUMEN}

Objetivo: identificar las acciones del equipo enfermería en la evaluación y manejo del dolor en el recién nacido. Método: Se trata de una Scoping review, realizada en agosto de 2016, mediante búsqueda en las bases de datos Latinoamericana y del Caribe en Ciencias de la Salud y Base de Datos de la Enfermería. Se seleccionaron ocho artículos que cumplieron los criterios de inclusión. Resultados: Se identificaron estudios transversales, exploratorios cualitativos y cuantitativos. Los resultados evidenciaron dos categorías, siendo ellas: "La percepción de los profesionales de enfermería frente al conocimiento del dolor neonatal, los medios de evaluación y la utilización de escalas de dolor" a la que fue posible comprobar que los profesionales de enfermería reconocen que los recién nacidos, los nacidos son capaces de sentir dolor, las formas de evaluar por medio de aspectos conductuales y fisiológicos y la existencia de escalas para medir dolor. Como segunda categoría "Medidas no farmacológicas utilizadas en el manejo del dolor neonatal" que contemplan la prescripción de fármacos y conducta de manejo / posicionamiento. Conclusión: Se concluye que aún hay una gran distancia entre el conocimiento teórico, el uso de escalas y la conducta práctica de los profesionales en relación a la evaluación y manejo del dolor neonatal.

Descriptores: Dolor, percepción del dolor, recién nacido, enfermería

\section{INTRODUÇÃO}

Nas últimas décadas, com o desenvolvimento científico e tecnológico observa-se, nas Unidades de Tratamento Intensivo Neonatais (UTIN), uma redução da mortalidade e uma maior expectativa para a sobrevida do Recém-Nascido (RN). Esta sobrevida, porém tem um custo, que inclui a dor, previsível nas terapias invasivas, mas pouco valorizada nos cuidados rotineiros. Os neonatos internados na UTIN, estão expostos a diversas intervenções, convivendo com inúmeros procedimentos agressivos, estressantes e dolorosos devido aos recursos tecnológicos da assistência. Estes procedimentos produzem risco a sua estabilidade, causando impacto no seu crescimento e desenvolvimento, que podem causar alterações fisiológicas de diversas ordens e inclusive sequelas neurológicas. Para isso, deve ser priorizado o cuidado especialmente focado na atenção integral e humanizada, visando qualidade de vida ao neonato. ${ }^{1,2,3}$

Neste contexto, a dor é definida pela Sociedade Internacional para o Estudo da Dor (IASP) como uma experiência sensitiva emocional desagradável, relacionada à lesão tecidual, como uma manifestação subjetiva, complexa, sendo 
multidimensional, que envolve mecanismos físicos, psíquicos e culturais. ${ }^{4} \mathrm{~A}$ dor neonatal foi negligenciada na prática clínica, durante anos, baseada no pressuposto de que o recém-nascido não possuía o sistema nervoso completamente formado, devido à mielinização incompleta de suas fibras nervosas, além da falta de memória para registrar os eventos dolorosos. A partir da década de oitenta, pesquisas trouxeram mudanças significativas para a neonatologia, principalmente no que se refere à dor, alterando a assistência; torna-se, então conhecido que em neonatos o mecanismo de modulação da experiência dolorosa é imaturo, o que limita sua capacidade para enfrentar a dor e o estresse. ${ }^{5}$

A dor neonatal é responsável por diversas complicações fisiológicas e comportamentais e pode gerar alterações como: o aumento da pressão arterial, a diminuição da saturação de oxigênio, alterações metabólicas e endócrinas (incluindo o catabolismo, hipermetabolismo e supressão da atividade da insulina com consequente hiperglicemia). $\mathrm{O}$ neonato pode apresentar-se suscetível a infecções e, também alterações na coagulação e na hemostasia. ${ }^{4}$

À dor persistente o neonato apresenta passividade, poucos movimentos corporais, face sem expressão, decréscimo da frequência cardíaca, variabilidade respiratória, além de um decréscimo do consumo de oxigênio. Com isso, torna-se necessário utilizar recursos que permitam minimizar os efeitos da hospitalização, onde o tempo de internação não deve se tornar uma experiência traumática ou uma interrupção no seu desenvolvimento. ${ }^{4}$

$\mathrm{O}$ enfermeiro e a equipe de enfermagem por serem profissionais responsáveis pelo maior período de acompanhamento do RN durante sua internação, assumem um papel relevante na observação criteriosa e identificação da ocorrência de sinais que traduzam a presença de dor. $\mathrm{O}$ reconhecimento e a aceitação da presença da dor por parte do cuidador é fundamental para a avaliação adequada. $^{2-4}$

Estudos de revisão do tipo escopo, que permitem um mapeamento amplo do conhecimento sobre esta temática não foram identificados na literatura. Nesta perspectiva, entender a relevância da percepção da dor no recém-nascido, devido à sua não verbalização, torna-se um fator determinante para que o período de internação seja menos doloroso e traumatizante possível, onde deve haver a interação e comunicação entre os membros da equipe de saúde, que passam a atender e a perceber a evolução da dor em cada paciente e verificar a resposta frente a terapia empregada. ${ }^{3}$ 
Embora existam as evidências que demonstram que a dor é um evento real e comum nesta população, e responsável por diversas complicações não somente em curto prazo, mas também em longo prazo, poucos profissionais avaliam a dor de modo sistematizado e empregam medidas preventivas e terapêuticas adequadas durante a internação destes pacientes. ${ }^{4}$

Ademais, o RN e lactentes não manifestarem verbalmente a ocorrência da dor, essa manifestação torna-se repetida e faz-se necessário que os profissionais envolvidos nos cuidados utilizem outros métodos para avaliá-la, baseados nas alterações comportamentais, fisiológicas e hormonais, que futuramente podem favorecer comprometimentos neurológicos, emocionais e cognitivos, até mesmo problemas psiquiátricos. ${ }^{2-4,5}$ Sendo assim, este artigo tem por objetivo identificar as ações da equipe enfermagem na avaliação e manejo da dor no recém-nascido.

\section{MÉTODO}

Trata-se de uma Scoping review, que seguiu o protocolo de identificação da questão de investigação e dos estudos relevantes; seleção desses estudos e a extração dos dados; agrupamento, resumo e relato dos resultados; consulta a especialistas (opcional) para síntese de produções. $^{6,7}$ A questão norteadora foi: $\mathrm{O}$ que se tem produzido na literatura científica frente as ações da equipe enfermagem na avaliação e manejo da dor no recémnascido?

Foi realizada busca na Biblioteca Virtual em Saúde (BVS) nas bases de dados Literatura Latino-Americana e do Caribe em Ciências da Saúde (LILACS) e Base de Dados da Enfermagem (BDENF). Para coleta de dados na BVS foi realizada a seguinte estratégia de busca: "DOR" AND "PERCEPÇÃO DA DOR”, AND, "RECÉM-NASCIDO” [Título, resumo, assunto]. Como critérios de inclusão foram selecionados estudos no recorte temporal de 2010 a 2016, que responderam à pergunta da pesquisa abordada, publicados com resumos disponíveis e acessados na íntegra pelo meio on-line, no idioma português. Foram excluídos artigos teóricos, revisões integrativas, narrativas e sistemáticas, relatos de experiência, editoriais, teses, dissertações, monografias, resumos e, documentos não condizentes com a temática abordada. Após a localização dos artigos, realizou-se a leitura dos resumos do material encontrado e, em seguida, a análise dos dados. A coleta ocorreu no mês de agosto de 2016 e o processo está apresentado na Figura 1. 
Figura 1 - Fluxograma da coleta de dados e seleção dos estudos que compõem a amostra. Santa Maria, 2017.
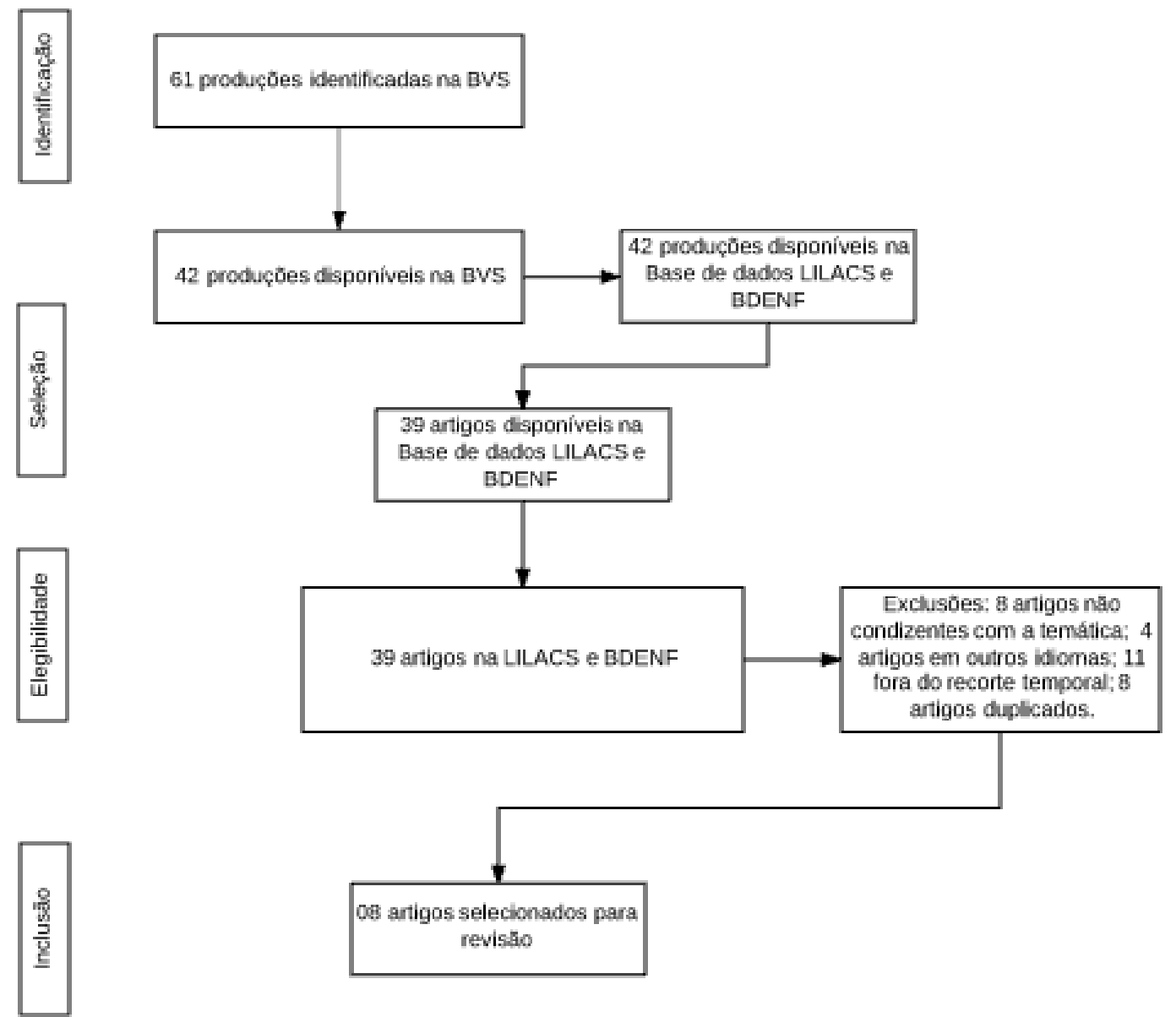

Fonte: Dados da pesquisa (2017)

A análise dos dados foi realizada por meio de cálculos de frequência simples e relativa. As atividades dos enfermeiros descritas nos artigos foram classificadas por similaridade e agrupadas em categorias, com utilização do programa NVivo, versão 10.

\section{RESULTADOS}

A estratégia de busca permitiu recuperar 61 registros. A leitura com análise dos títulos e resumos resultou na seleção de
39 publicações para leitura na íntegra. A partir da leitura do texto integral, um total de oito publicações responderam a questões norteadora da revisão de escopo e caracterizam a amostra desta busca teórica. A Figura 1 representa o processo de seleção dos artigos, com base na recomendação PRISMA. A Tabela 1 apresenta os artigos, conforme autores, ano de publicação, objetivo principais resultados, conclusões e palavras-chaves. 
Tabela 1 - Descrição dos artigos incluídos na revisão. Santa Maria, RS, Brasil, 2017.

\begin{tabular}{|c|c|c|c|}
\hline $\begin{array}{l}\text { Autores e } \\
\text { ano }\end{array}$ & Objetivo & Principais resultados & Conclusões \\
\hline $\begin{array}{l}\text { Veronez M, } \\
\text { Corrêa } \\
\text { DAM, } \\
\text { 2010. }^{8}\end{array}$ & $\begin{array}{l}\text { Descrever a percepção } \\
\text { dos profissionais de } \\
\text { enfermagem que atuam } \\
\text { em UTIN sobre a dor } \\
\text { no recém-nascido. }\end{array}$ & $\begin{array}{l}\text { Os resultados envolvem reconhecimento da dor } \\
\text { pelos participantes, a utilização de medidas } \\
\text { farmacológicas e não-farmacológicas, a punção } \\
\text { venosa como procedimento mais doloroso e a } \\
\text { necessidade de reconhecimento e avaliação para } \\
\text { intervenções no fenômeno. }\end{array}$ & $\begin{array}{l}\text { É emergencial a implantação de um } \\
\text { protocolo de avaliação da dor na } \\
\text { unidade e treinamento da equipe } \\
\text { envolvida, contribuindo para a } \\
\text { humanização da assistência. }\end{array}$ \\
\hline
\end{tabular}

Unidades de registo

- Dor;

- Fármacos;

- Medidas

farmacológicas;

- Procedimentos invasivos:

- Ausência de protocolos

\begin{tabular}{|c|c|c|c|c|}
\hline $\begin{array}{l}\text { Bemfica } \\
\text { AF, Fialho } \\
\text { FA, Dias } \\
\text { IMAV, } \\
\text { Amorim } \\
\text { TM, 2013. }\end{array}$ & $\begin{array}{l}\text { Discutir a percepção da } \\
\text { equipe de enfermagem } \\
\text { em relação à dor do } \\
\text { neonato identificando } \\
\text { as atitudes destes } \\
\text { profissionais frente ao } \\
\text { recém-nascido com dor } \\
\text { na UTIN. }\end{array}$ & $\begin{array}{l}\text { Os profissionais apesar de identificarem a dor do } \\
\text { recém-nascido, vinculam o tratamento como uma } \\
\text { ação dependente da prescrição médica. }\end{array}$ & $\begin{array}{l}\text { O aprofundado deste tema em } \\
\text { programas de educação permanente } \\
\text { para que haja uma maior } \\
\text { sensibilização por parte dos } \\
\text { profissionais. }\end{array}$ & $\begin{array}{l}\text { - Dor; } \\
\text { - } \quad \text { Capacitação da equipe; } \\
\text { - } \quad \text { Avaliação por escala. }\end{array}$ \\
\hline $\begin{array}{l}\text { Caetano } \\
\text { EA, Lemos } \\
\text { NRF, } \\
\text { Cordeiro } \\
\text { SM, Pereira } \\
\text { FMV, } \\
\text { Moreira } \\
\text { DS, } \\
\text { Buchhorn } \\
\text { SMM, } \\
\text { 2013.10 }^{\text {. }}\end{array}$ & $\begin{array}{l}\text { Descrever as formas de } \\
\text { avaliação de dor do } \\
\text { recém-nascido } \\
\text { utilizadas pela equipe } \\
\text { de enfermagem e } \\
\text { analisar a prática da } \\
\text { enfermagem quanto ao } \\
\text { manejo da dor do } \\
\text { neonato. }\end{array}$ & $\begin{array}{l}\text { Os entrevistados acreditam que o recém-nascido é } \\
\text { capaz de sentir dor. Porém não há utilização de } \\
\text { escalas de avaliação da dor padronizadas nas } \\
\text { instituições. }\end{array}$ & $\begin{array}{l}\text { Assim, há necessidade de capacitar } \\
\text { os profissionais, contribuindo para a } \\
\text { avaliação e o manejo da dor, e } \\
\text { promovendo o cuidado integral ao } \\
\text { neonato. }\end{array}$ & $\begin{array}{l}\text { - Necessidade de escalas de } \\
\text { dor; } \\
\text { - } \quad \text { Protocolos para avaliar a } \\
\text { dor; } \\
\text { - Educação continuada. }\end{array}$ \\
\hline
\end{tabular}




\begin{tabular}{|c|c|c|c|c|}
\hline $\begin{array}{l}\text { Amaral JB, } \\
\text { Resende } \\
\text { TA, Contim } \\
\text { D, } \\
\text { Barichello } \\
\text { E, 2014. }\end{array}$ & $\begin{array}{l}\text { Identificar as formas de } \\
\text { avaliação e manejo da } \\
\text { dor do recém-nascido } \\
\text { prematuro. }\end{array}$ & $\begin{array}{l}\text { Todos os profissionais concordaram sobre a } \\
\text { capacidade do RN de sentir dor. O choro, } 42(100 \%) \text {; } \\
\text { face, } 40(95,2 \%) \text {; e frequência cardíaca, } 39(92,8 \%) \text {, } \\
\text { foram os parâmetros de avaliação mais } \\
\text { mencionados. }\end{array}$ & $\begin{array}{l}\text { Utilizam medidas não } \\
\text { farmacológicas. Não há utilização } \\
\text { de escalas. A equipe acredita na } \\
\text { necessidade de capacitação sobre o } \\
\text { tema. }\end{array}$ & $\begin{array}{ll}\text { - } & \text { Choro; } \\
\text { - } & \text { Mimica facial; } \\
\text { - } & \text { Escalas para medição; } \\
\text { - } & \text { Educação continuada; } \\
\text { - } & \text { Medidas não } \\
& \text { farmacológicas. }\end{array}$ \\
\hline $\begin{array}{l}\text { Monfrim } \\
\text { XM, } \\
\text { Saraiva LA, } \\
\text { Moraes CL, } \\
\text { Viegas AC, } \\
\text { 2015.12 }\end{array}$ & $\begin{array}{l}\text { Conhecer a percepção } \\
\text { de enfermeiros com } \\
\text { relação à utilização de } \\
\text { um instrumento para } \\
\text { avaliação da dor em } \\
\text { neonatos prematuros. }\end{array}$ & $\begin{array}{l}\text { Foram abordadas categorias como: Escala para } \\
\text { avaliação da dor nos RN prematuros: percepção dos } \\
\text { enfermeiros e Escala de dor e o prematuro: sua } \\
\text { implementação. Percebe-se que os enfermeiros } \\
\text { desconhecem as novas tecnologias utilizadas para } \\
\text { mensurar a dor. }\end{array}$ & $\begin{array}{l}\text { A capacitação da equipe é uma } \\
\text { estratégia para o entendimento } \\
\text { acerca da dor, já que todos } \\
\text { conseguem percebê-la. }\end{array}$ & $\begin{array}{l}\text { - } \quad \text { Escalas de dor; } \\
\text { - Desconhecimento de } \\
\text { como medir a dor; } \\
\text { - Capacitação da equipe; } \\
\text { - Percepção de dor. }\end{array}$ \\
\hline
\end{tabular}

\begin{tabular}{ll}
\hline Silva GM, & Verificar o \\
Figueiredo & conhecimento que os \\
MGS, & enfermeiros de unidade \\
Kameo SY, de terapia intensiva \\
Oliveira & neonatal possuem sobre \\
FM, Santos & a dor no recém-nascido. \\
AD, 2015. &
\end{tabular}

o Emergiram seis categorias: percepção pelos

enfermeiros quanto à interação mãe, recém-nascido,

família; conceito e reconhecimento da dor;

conhecimento dos enfermeiros sobre escalas de dor;

atitudes de enfermeiros no alívio da dor; percepções

dos enfermeiros sobre a humanização na unidade de

terapia intensiva neonatal.
Os profissionais executam medidas de alívio da dor e promovem interação com a família, porém apresentam dificuldades na implantação de protocolos que viabilizem o manejo mais adequado da dor.
- Dor

- Família

- Escalas;

- Condutas para manejo da dor;

- Protocolos.

\begin{tabular}{|c|c|c|c|c|}
\hline $\begin{array}{l}\text { Costa } \\
\text { Alves } \\
\text { Dames } \\
\text { et } \\
2016 .{ }^{14}\end{array}$ & $\begin{array}{l}\text { Analisar a percepção } \\
\text { dos enfermeiros acerca } \\
\text { da clínica da dor no } \\
\text { neonato na unidade de } \\
\text { terapia intensiva } \\
\text { neonatal. }\end{array}$ & $\begin{array}{l}\text { Avaliam a dor neonatal pelos sinais fisiológicos e } \\
\text { comportamentais. Não é citado método de avaliação. }\end{array}$ & $\begin{array}{l}\text { Necessidade de instituir a utilização } \\
\text { de protocolos e escalas para a } \\
\text { avaliação dos indicadores de dor } \\
\text { neonatal na prática. }\end{array}$ & $\begin{array}{l}\text { - } \text { Sinais fisiológicos; } \\
\text { - } \quad \text { Comportamento do RN; } \\
\text { - } \quad \text { Escala de dor; } \\
\text { - } \quad \text { Protocolos para medir } \\
\text { dor. }\end{array}$ \\
\hline
\end{tabular}




\begin{tabular}{|c|c|c|c|c|}
\hline $\begin{array}{l}\text { Silva PC, } \\
\text { Marinho } \\
\text { EFC, } \\
\text { Santos } \\
\text { LOS, } \\
\text { 2016. }^{15}\end{array}$ & $\begin{array}{l}\text { Analisar a percepção } \\
\text { dos profissionais de } \\
\text { saúde sobre a dor em } \\
\text { prematuro, a forma de } \\
\text { avaliação, uso de } \\
\text { escalas e protocolo para } \\
\text { tratamento. }\end{array}$ & $\begin{array}{l}\text { Os profissionais de saúde envolvidos na pesquisa } \\
\text { acreditam que o recém-nascido sente dor, porém } \\
\text { encontram dificuldades em avaliá-la. }\end{array}$ & $\begin{array}{l}\text { Há necessidade de uma educação } \\
\text { permanente desta equipe } \\
\text { multiprofissional em relação à dor } \\
\text { no período neonatal, destacando as } \\
\text { escalas disponíveis e a utilização de } \\
\text { protocolos para esta avaliação. }\end{array}$ & $\begin{array}{ll}\text { - } & \text { Dificuldade de medir; } \\
\text { - } & \text { Educação permanente; } \\
\text { - } & \text { Equipes } \\
\text { - } & \text { multiprofissionais; } \\
\text { - } & \text { Escalas; } \\
\text { Protocolos. }\end{array}$ \\
\hline
\end{tabular}

Fonte: Dados da pesquisa (2017) 
Nos estudos selecionados as pesquisas ocorreram por meio de entrevistas direcionadas aos profissionais de enfermagem, abrangendo qual o conhecimento e percepção sobre a dor no $\mathrm{RN}$, sobre o uso ou não de instrumentos para avaliar a dor e também estratégias utilizadas para seu manejo. Desses 37,5\% elaboraram entrevistas, somente, com enfermeiros, $50 \%$ dos estudos foram a equipe de enfermagem (enfermeiros, técnicos e auxiliares de enfermagem) e $12,5 \%$ com entrevistas que englobavam uma equipe multidisciplinar (enfermeiros, fisioterapeutas, médicos e psicólogos).

Em relação ao delineamento dos artigos selecionados, foram identificados cinco $(62,5 \%)$ exploratório qualitativo, dois (25\%) quantitativo transversal e um (12,5\%) exploratório quantitativo.

Referente ao ano de publicação dos artigos observou-se que dois estudos (25\%) foram publicados no ano de 2016, dois (25\%) em 2015, um (12,5\%) em 2014, dois (25\%) em 2013 e um (12,5\%) em 2010.

Para fins de discussão emergiram três categorias, sendo elas: "A percepção dos profissionais de enfermagem frente ao conhecimento da dor neonatal, os meios de avaliação e a utilização de escalas de dor" e "Medidas não farmacológicas utilizadas no manejo da dor neonatal".

\section{DISCUSSÃO}

Na primeira categoria é identificada como é a percepção dos profissionais de enfermagem frente ao conhecimento da dor neonatal, os meios de avaliação e a utilização de escalas de dor. Foi possível comprovar por meio da literatura, que os profissionais de enfermagem reconhecem que os recém-nascidos são capazes de sentir dor. Esse resultado, foi transmitido por eles através de suas experiências da rotina de trabalho, de suas crenças e valores. ${ }^{8-15}$

Apesar de todos os entrevistados acreditarem que o $\mathrm{RN}$ sente dor, as respostas de dois estudos divergem, no que diz respeito ao neonato ser mais sensível à dor do que os adultos ou crianças mais velhas. Os entrevistados acreditam que a imaturidade do sistema nervoso do $\mathrm{RN}$ faz com que esse seja mais sensível a dor $^{8}$ diferente do apresentado em estudo ${ }^{9}$ cujas respostas indicaram não existir diferença na sensibilidade da dor entre as faixas etárias. Embora a literatura afirme que a intensidade com que o neonato percebe a dor, é maior do que das outras faixas etárias em razão da imaturidade dos seus mecanismos de controle inibitório da dor pois este só se torna funcional após as primeiras semanas depois do nascimento, e isto o torna mais sensível. ${ }^{16}$

Assim o RN, principalmente o prétermo, não consegue modular de forma eficaz os estímulos dolorosos, o que limita 
sua capacidade de enfrentar a dor e o estresse. Essa imaturidade na modulação, ao invés de inibir, acaba por ampliar a sensação dolorosa e desencadear respostas generalizadas e exageradas, principalmente no RN pré-termo. ${ }^{2,3,8}$

A dor no período neonatal é identificada pelos entrevistados ${ }^{8}$ 11, 13-15 através da observação de aspectos comportamentais e fisiológicos. Dessa forma, são utilizados parâmetros, dentre os quais os mais citados foram: o choro, a mímica facial e o movimento corporal. Em $75 \%$ dos estudos, o choro é o parâmetro comportamental mais relatado como forma de identificar se o RN está com dor, sendo que, parte dos entrevistados relatam identificar a diferença entre um choro de dor e o choro por outro desconforto. A emissão do choro é tensa e estridente, com tonalidade mais aguda e com aumento da duração. Normalmente o choro possui determinado padrão melódico e uma frequência de 80 decibéis. $^{3}$

Na presença de estímulos dolorosos o choro sofre alterações delicadas como o prolongamento da fase expiratória, perda do padrão melódico, aumento da duração e a tonalidade mais aguda. ${ }^{3} \mathrm{O}$ choro de dor é estridente e possui melodia ascendentedescendente, longa duração e uma frequência de 530 Hertz. ${ }^{17}$

Assim, o choro é definido como um método primário, destacando que durante procedimentos dolorosos, $50 \%$ dos bebês não choram. Ainda, pode indicar fome e/ou

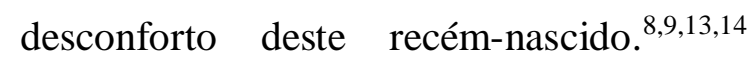
Portanto, como método isolado, o choro, é questionável e pouco específico para ser utilizado como avaliação da dor na prática clínica. $^{18}$

A mímica facial foi abordada nos estudos $^{9-11,13,14}$ como sendo um dos parâmetros mais empregados no estudo da dor no recém-nascido. Destaca-se fronte saliente, fenda palpebral estreitada, sulco naso-labial aprofundado, lábios entreabertos, boca estirada, tremor do mento e língua tensa. Método bastante útil, específico na rotina para avaliação da dor, porém, as alterações da face não trazem informações quanto a qualidade e intensidade da dor. Além disso, sabe-se que ocorrem alterações aos estímulos dolorosos agudos, mas não se conhece diante de um estímulo prolongado e repetitivo. ${ }^{3}$

Quanto ao movimento corporal $^{11,13,15}$ citado como uma percepção da dor por meio de contrações musculares, movimentos contínuos de braços e pernas, irritabilidade. A facilidade em identificar a movimentação do RN no dia-a-dia das UTIN, juntamente com a relação entre o estímulo doloroso e alteração nesse comportamento, além da existência de movimentos específicos associados à sensação dolorosa explicam e comprovam a importância da observação da resposta 
motora na avaliação da dor nesses pacientes. $^{17}$

As alterações fisiológicas também são observadas e atribuídas ao processo doloroso, porém citadas em menor proporção que as alterações comportamentais. As alterações fisiológicas mais relatadas foram $\mathrm{o}$ aumento $\mathrm{da}$ frequência cardíaca e frequência respiratória e diminuição da saturação de oxigênio. ${ }^{8-11,13-15}$

Os parâmetros que podem sugerir dor são o aumento da frequência cardíaca e frequência respiratória, diminuição da saturação de oxigênio, alterações da pressão arterial e intracraniana, dilatação das pupilas, tremores, bem como diminuição da motilidade gástrica, cianose e sudorese palmar, bem como alterações metabólicas e endócrinas, com aumento de hormônios como cortisol, noradrenalina e adrenalina. 19,20

Por isso, ainda podem ocorrem episódios de apneia, utilização de reservas de glicose, alterações do fluxo sanguíneo cerebral e hemorragia ventricular, congestão venosa e hipóxia. ${ }^{17,19,20}$ Essas alterações afetam o equilíbrio homeostático do $\mathrm{RN}$, que por suas condições clínicas já é instável e precário. Essas alterações fisiológicas, apesar da facilidade de verificação e da boa aplicabilidade, são pouco específicas para a dor, pois podem ocorrer devido à própria patologia do $\mathrm{RN}$, ou mesmo a situações apenas desconfortáveis, mas não dolorosas. ${ }^{17}$

As alterações fisiológicas podem ser usadas na avaliação, qualificação e quantificação do estímulo doloroso, porém, somente quando inseridas no contexto em que $\mathrm{o}$ neonato se encontra, e ainda acompanhadas por métodos comportamentais ou multidimensionais, escalas de avaliação de dor. ${ }^{21}$

A criação de instrumentos de avaliação de dor foi um grande avanço para avaliação de RN. Diversos métodos têm sido publicados e são válidos para uso clínico nos últimos anos, trazendo benefícios no tratamento nesta faixa etária. As escalas de dor, tornam mais objetiva a avaliação do RN, de forma a garantir a intervenção mais segura. ${ }^{22}$

Quando questionados sobre a existência de escalas que avaliem a dor no $\mathrm{RN}$, nenhum dos artigos analisados apresentou utilização e avaliação significativas das escalas de dor. ${ }^{8-15}$ Demonstrando uma fragilidade, pois os instrumentos que avaliam a dor no RN estão disponíveis e vêm sendo descritos na literatura desde o fim da década de 80. Não há política institucional, nem mesmo uso de protocolo assistencial para a avaliação da dor em recém-nascidos internados em UTIN. $^{23}$

A escala de avaliação da dor NIPS, apresentou o maior relato de conhecimento 
de escalas, foi a mais citada, com 70,96\% dos profissionais. Porém na prática, as respostas apresentaram-se não específicas a utilização de algum tipo de escala. ${ }^{11}$ Ainda, dos 42 profissionais que participaram de um estudo $^{10}$, um utilizava a escala NIPS para avaliar a dor. Quando citam a NIPS, é demonstrado que os profissionais reconhecem a dor por parâmetros comportamentais e fisiológicos em conjunto, já que na avaliação os parâmetros isolados não são adequados. ${ }^{10,22}$

Os enfermeiros referiram conhecer algumas escalas de avaliação da dor como a NIPS (Neonatal Infant Pain Scale), NFCS (Neonatal Facial Condin System) e PIPP (Premature Infant Pain Profile), porém não existe escala de dor implantada na rotina da unidade. ${ }^{13}$ As escalas NIPS, NFCS e PIPP foram as mais encontradas na literatura relacionadas à avaliação de um procedimento específico doloroso e as escalas NIPS, NFCS e "faces" incorporadas na rotina da UTIN, há predomínio da NIPS nas duas formas de utilização mencionadas. $^{24}$

Ações direcionadas a aplicação de uma escala por enfermeiros foram identificadas durante o período de duas semanas a prematuros internados. Destacase que os profissionais do estudo desconhecem sobre os instrumentos de avaliação da dor, e o desafio para a enfermagem não é a percepção e sim a capacitação da equipe, uma vez que, não era utilizado nenhum instrumento na unidade do estudo. ${ }^{12}$

$\mathrm{Na}$ segunda categoria abordam-se condutas não farmacológicas direcionadas ao manejo da dor no RN. A maioria dos entrevistados utiliza estratégias próprias, porém sem medida preventiva. ${ }^{8-10,13,15}$ As autoras $^{8}$ acreditam que a baixa taxa de intervenção se deve ao fato deste tema ser pouco explorado durante a formação profissional. A medida preventiva sempre antes do previsto, pode evitar possíveis sequelas clínicas, fisiológicas e/ou psicológicas, a curto e a longo prazo. ${ }^{25}$

Apesar de terem sido citadas medidas não farmacológicas pelos profissionais de nível superior, estes ainda são dependentes da prescrição médica, com dificuldade de intervir com outros métodos além da sedação e analgesia. ${ }^{9}$

No estudos ${ }^{10,11}$ foram destacados como conduta dos profissionais frente aos sinais de dor do neonato, as intervenções de manuseio/posicionamento, sucção não nutritiva.

A conduta manuseio/posicionamento com determinada contenção pode ser considerada suporte postural, o neonato sente-se aconchegado e sua organização é preservada, uma vez que a contenção facilitada determina limites ao RN tal como ocorria no ambiente uterino. ${ }^{1}$ Essa intervenção age no SNC, uma vez que envia 
uma cascata de estímulos que competem com a sensação dolorosa modulando a percepção da nocicepção. ${ }^{1,26}$

Dentre as intervenções nãofarmacológicas para prevenir ou tratar a dor, as mais citadas foram a utilização de glicose ou sucção não nutritiva, promoção de ambiente confortável, manuseio mínimo e o método Canguru. Todos métodos relatados já foram testados e comprovados o efeito redutor na dor. ${ }^{13}$

A utilização de glicose ou sucção não nutritiva também foi citada como medida não farmacológica antes e durante os procedimentos dolorosos. ${ }^{13,15}$ Sua eficácia no alívio da dor neonatal já foi comprovada, principalmente no que diz respeito à glicose e à sacarose. Diminui o tempo de choro, aumento da oxigenação, diminuição da frequência e gasto energético, promovendo descanso e analgesia. $^{22,23}$

\section{CONSIDERAÇÕES FINAIS}

Os resultados deste estudo permitiram a identificação das ações da equipe enfermagem na avaliação e manejo da dor no recém-nascido. Demonstraram que apesar de os profissionais de enfermagem reconhecerem a dor do RN, ainda há uma grande distância entre o conhecimento teórico, o uso de escalas e a conduta prática dos profissionais em relação à avaliação e manejo da dor neonatal. Portanto, as evidências demonstram que a avaliação da dor através de escalas não é uma realidade, assim como também, não existe uma sistematização para essa assistência do RN no manejo da dor.

Como limitação do estudo, considera-se a delimitação do período de busca em seis anos que pode ter desconsiderado artigos que contribuiriam para agregar resultados a questão de pesquisa. Assim, é necessária a capacitação com educação continuada da equipe de enfermagem, com conhecimentos específicos e aprofundados para o conhecimento e percepção da dor com destaque para a utilização de tecnologias disponíveis e a implementação de protocolos para a avaliação da dor como prática obrigatória que favorece uma assistência integral ao neonato.

\section{REFERÊNCIAS}

1. Ministério da Saúde (Brasil). Atenção à saúde do recém-nascido: guia para os profissionais de saúde. Brasília, DF: Ministério da Saúde; 2012. (Cuidados com o recém-nascido pré- termo; v. 4). 2 Mota LA, Sá FE, Frota MA, Estudo comparativo do desenvolvimento sensório motor de recém-nascidos prematuros da unidade de terapia intensiva neonatal e do método canguru. Rev Bras Promoç Saúde [Internet]. 2005 [citado em 24 jan 2019]; 18(4):191-98. Disponível em: http://www.redalyc.org/html/408/4081840 6/ 3 Guinsburg R, Leslie ATS, Covolan L. Consequência da dor repetida ou 
persistente no período neonatal.

ComCiência [Internet]. 2007 [citado em 20 jan 2019]; (87). Disponível em:

http://www.com19encia.br/comciencia/han dler.php?section $=8 \&$ edicao $=24 \&$ id $=269$

4 Bueno M, Kimura AF, Pimenta CAM.

Avaliação da dor em recém-nascidos submetidos à cirurgia cardíaca. Acta Paul Enferm. [Internet]. 2007 [citado em 20 jan 2019]; 20(4):428-33. Disponível em: http://www2.unifesp.br/acta/pdf/v20/n4/v2 0n4a7.pdf

5 Lago CW, Ferreira GG, Lima JB, Ribeiro SFF, Santos VPV. Avaliação e manejo da dor neonatal no contexto da unidade de terapia intensiva neonatal [Monografia]. Brasília, DF: Universidade Paulista; 2007.

6 Arksey H, O’Malley L. Scoping studies: towards a methodological framework. Int J Soc Res Methodol. [Internet]. 2005 [citado em 24 jan 2019]; 8(1):19-32. Disponível em:

https://www.tandfonline.com/doi/pdf/10.10 80/1364557032000119616?needAccess $=\operatorname{tr}$ ue

7 Levac D, Colquhoun H, O’Brien KK.

Scoping studies: advancing the methodology. Implement Sci. [Internet]. 2010 [citado em 24 jan 2019]; 5(69):1-9. Disponível em:

https://www.ncbi.nlm.nih.gov/pmc/articles /PMC2954944/

8 Veronez M, Corrêa DAM. A dor e o recém-nascido de risco: percepção dos profissionais de enfermagem. Cogitare Enferm. [Internet]. 2010 [citado em 20 jan 2019]; 15(2):263-70. Disponível em: https://www.revistas.ufpr.br/cogitare/articl e/download/17859/11652

9 Alves FB, Fialho FA, Dias IMAV, Amorim TM, Salvador M. Dor neonatal: a percepção da equipe de enfermagem na unidade de terapia intensiva neonatal. Rev Cuid. (Bucaramanga). [Internet]. 2013 [citado em 20 jan 2019]; 4(1):510-15. Disponível em: http://www.scielo.org.co/pdf/cuid/v4n1/v4 n1a11.pdf
10 Caetano EA, Lemos NRF, Cordeiro SM, Pereira FMV, Moreira DS, Marques SM. The new-born with pain: the role of the nursing team. Esc. Anna Nery Rev Enferm. [Internet]. 2013 [citado em 20 jan 20190]; 17(3):439-45. Disponível em: http://www.scielo.br/pdf/ean/v17n3/14148145-ean-17-03-0439.pdf

11 Amaral JB, Resende TA, Contim D, Barichello E. Equipe de enfermagem diante da dor do recém-nascido pré-termo. Esc. Anna Nery Rev Enferm. [Internet]. 2014 [citado em 24 jan 2019]; 18(2):24146. Disponível em: http://www.scielo.br/pdf/ean/v18n2/14148145-ean-18-02-0241.pdf

12 Monfrim XM, Saraiva LA, Moraes CL, Viegas AC. Escala de avaliação da dor: percepção dos enfermeiros em uma unidade de terapia intensiva neonatal. Rev Enferm UFSM. [Internet]. 2015[citado em 24 jan 2019]; 5(1):12-22. Disponível em: https://periodicos.ufsm.br/reufsm/article/vi ew/15049/pdf

13 Silva GM, Figueiredo MGS, Kameo

SY, Oliveira FM, Santos AD.

Conhecimento das enfermeiras atuantes em unidade de terapia intensiva frente a dor no recém-nascido pré-termo. Rev Iberoam Educ Invest Enferm. [Internet]. 2015 [citado em 20 jan 2019]; 5(1):47-55. Disponível em:

https://www.enfermeria21.com/revistas/ala defe/articulo/150/

14 Costa KF, Alves VH, Dames LJP, Rodrigues DP, Barbosa MTSR, Souza $\mathrm{RRB}$, et al. Manejo clínico da dor no recém-nascido: percepção de enfermeiros da unidade de terapia intensiva neonatal. Rev Pesqui Cuid Fundam. [Internet]. 2016 [citado em 20 jan 2019]; 8(1):3758-69.

Disponível em:

http://www.seer.unirio.br/index.php/cuidad ofundamental/article/viewFile/3950/pdf_1 786

15 Silva PC, Marinho EFC, Santos LOS. A percepção dos profissionais de saúde sobre a dor em prematuros. Diálogos \& Ciências, [Internet]. 2016 [citado em 20 jan 2019]; 36(16):39-51. Disponível em: 
https://periodicos.ftc.br/index.php/dialogos /article/view/18/pdf_03

16 Guinsburg R. Avaliação e tratamento da dor no recém-nascido. J Pediatr (Rio J.), [Internet]. 1999 [citado em 20 jan 2018]; 75(3):149-60. Disponível em: http://www.jped.com.br/conteudo/99-7503-149/port.pdf

17 Guinsburg R, Arias MCC. A linguagem da dor no recém-nascido: documento científico do Departamento de Neonatologia, Sociedade Brasileira de Pediatria [Internet]. São Paulo: SBP; 2010 [citado em 14 jul 2019]. Disponível em: http://www.sbp.com.br/pdfs/doc_linguage m-da-dor-out2010.pdf

18 Oliveira RM, Silva AVS, Chaves EMC, Sales NC. Avaliação comportamental e fisiológica da dor em recém-nascidos pelos profissionais de enfermagem. REME Rev Min Enferm. [Internet]. 2010[citado em 20 jan 2019]; 14(1):19-24. Disponível em: http://www.reme.org.br/artigo/detalhes/82 19 Barbosa SMM, Santos E, Zapata A, Sapienza AJ, et al. A dor no recémnascido: prevenção e terapêutica. RevDor [Internet]. 2000 [citado em 24 jan 2019]; 2(2):26-35. Disponível em: https://www.apeddor.org/index.php/publicacoes/artigos 20 Gaíva MAM, Dias NS. Dor no recémnascido: percepção de profissionais de saúde de um hospital universitário. Rev Paul Enferm. [Internet]. 2002 [citado em 24 jan 2019]; 21(3):234-9. Disponível em: http://www.scielo.br/scielo.php?script=sci _issues\&pid=0080-6234\&lng=en 21 Guimarães ALO, Vieira MRR. Conhecimento e atitudes da enfermagem de uma unidade neonatal em relação à dor no recém-nascido. Arq Bras Ciênc Saúde. [Internet]. 2008 [citado em 24 jan 2019]; 15(1):9-12. Disponível em:

http://repositorioracs.famerp.br/racs_ol/vol-151/IIIIDDDD220.pdf 22 Silva TP, Silva LJ. Escalas de avaliação da dor utilizadas no recém-nascido: revisão sistemática. Acta Med Port. [Internet]. 2010 [citado em 24 jan 2019]; 23 (3):437-
54. Disponível em: https://www.actamedicaportuguesa.com/re vista/index.php/amp/article/view/635/319 23 Santos LM, Pereira MP, Santos LFN, Santana RCB. Avaliação da dor no recémnascido prematuro em unidade de Terapia Intensiva. Rev Bras Enferm. [Internet]. 2012 [citado em 24 jan 2019]; 65(1):27-33. Disponível em: http://www.scielo.br/pdf/reben/v65n1/04.p df

24 Presbytero R, Costa MLV, Santos RCS. Os enfermeiros da unidade neonatal frente ao recém-nascido com dor. Rev Rene [Internet]. 2010 [citado em 21 mai 2010]; 11(1):125-32. Disponível em: https://www.redalyc.org/articulo.oa?id=32 4027969012

25 Medeiros MD, Madeira LM. Prevenção e tratamento da dor do recém-nascido em terapia intensiva neonatal. REME Rev Min Enferm. [Internet]. 2006 [citado em 24 jan 2019]; 10(2):118-24. Disponível em: http://www.reme.org.br/artigo/detalhes/395 26 Aquino FM, Christoffel MM. Dor neonatal: medidas não-farmacológicas utilizadas pela equipe de enfermagem. Rev Rene [Internet]. 2010 [citado em 21 maio 2010]; 11(N Esp):169-77. Disponível em: http://www.periodicos.ufc.br/rene/article/vi ew/4701/3496

RECEBIDO: 01/11/2017

APROVADO: $13 / 08 / 2018$

PUBLICADO: $12 / 2019$ 